\title{
Infertility in a Sahiwal Bull due to Bilateral Testicular Fibrosis
}

\author{
Bilawal Singh $^{1 *}$, Navgeet Singh ${ }^{2}$, Ajeet Kumar ${ }^{2}$, S.S. Dhindsa ${ }^{2}$ and Khushpreet Singh ${ }^{1}$ \\ ${ }^{1}$ Department of Veterinary and Animal Husbandry Extension Education, Guru Angad Dev \\ Veterinary and Animal Sciences University, Ludhiana, India-141004 \\ ${ }^{2}$ Department of Veterinary Gynaecology and Obstetrics, Guru Angad Dev Veterinary and \\ Animal Sciences University, Ludhiana, India-141004 \\ *Corresponding author
}

\section{A B S T R A C T}

\begin{tabular}{|l|}
\hline K e y w o r d s \\
Infertility, Sahiwal \\
bull, Testicular \\
fibrosis, \\
Ultrasonography \\
\hline Article Info \\
\hline $\begin{array}{l}\text { Accepted: } \\
17 \text { April } 2019 \\
\text { Available Online: } \\
\text { 10 May } 2019\end{array}$ \\
\hline
\end{tabular}

\section{Introduction}

Routine breeding soundness evaluation of bulls is quite an important tool to evaluate the potential bulls for their inculcation in the breeding programs and to point out any abnormality, especially in the reproductive system. The protocol for evaluation includes history of bull, physical examination and semen analysis. However, ultrasonography is an important diagnostic tool to pin point the exact etiologies associated with reproductive problems such as testicular fibrosis (Barth et al., 2008). Testicular fibrosis refers to the formation of excessive connective tissue in the testicle. Moreover, an increase in the sperm abnormalities has been found associated with the changes in echogenicity induced by scrotal insulation in bulls (Arteaga et al., 2005). Ultrasonographic diagnosis of testicular fibrosis is confirmed by the presence of variable number of hyperechoic foci of variable size. The present case report records similar echogenic changes in ultrasonography caused by bilateral testicular fibrosis.

\section{History and Observations}

An eight year old, otherwise healthy Sahiwal cattle bull (Fig. 1) was presented to the University Veterinary Hospital with the chief 
complaint of infertility for last six months in spite of several mating attempts. Semen analysis performed on periodic ejaculates collected from animal at field level revealed watery consistency along with oligospermia. Screening of bull for reproductive diseases and chronic diseases causing reproductive problems was also done periodically, with no apparent illness. Semen analysis performed on the ejaculate collected after presentation of the animal showed very less number of spermatozoa with decreased progressive motility and abnormal morphology. It was decided to perform ultrasonography of the reproductive organs, chiefly testes to diagnose the root cause of the ailment (Fig. 2). Ultrasonography of the testes was suggestive of multiple extremely hyperechoic patches, distributed uniformly in the entire testicular parenchyma, thus pointing towards the etiology being testicular fibrosis.

\section{Treatment and Discussion}

Fibrotic lesions were found extending from rete testes towards the periphery of the testes, thus covering the entire testicular parenchyma. Entire tubules in both the testes were destroyed with replacement by fibrotic tissue (Fig. 3). As testicular fibrosis is an irreversible event, no treatment is recommended because the fibrotic lesions cannot be reversed. The animal with bilateral testicular fibrosis is declared unfit for reproduction and culling of the animal from breeding programs is advisable.

The etiology of testicular destruction and fibrosis is variable involving abnormal development of seminiferous tubules to retetestes connections, infectious diseases affecting testes, abnormal thermoregulation mechanism and trauma (Barth et al., 2008). In young bulls, developmental changes of the testes might be involved in the testicular fibrotic lesions. Infectious agents can cause inflammation of the blood vessels of the testes, thus disturbing the thermoregulation, which can cause tissue necrosis and form fibrotic foci (McEntee, 1990). Various viral infections can also target the sertoli cells and germinal epithelium resulting in tubule damage and fibrous tissue infilteration (Ramirez-Mendoza et al., 1997). Moreover, trauma to the testes caused by blow injury due to kicking or butting has also been proposed as a possible cause of testicular fibrosis (McEntee, 1990).

Fig.1 Sahiwal bull which was presented at University Hospital for infertility issue

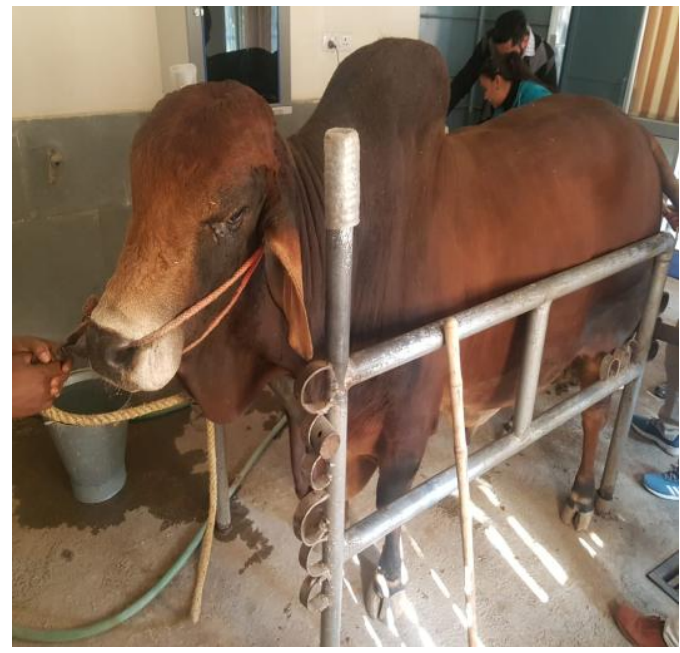


Fig.2 Testicular ultrasonography of bull

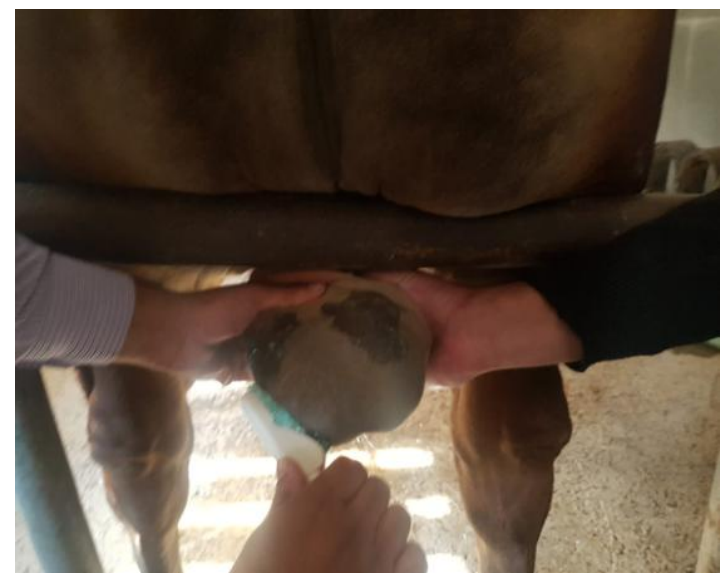

Fig.3 Ultrasonographic images showing variable number of extensively hyperechoic fibrotic foci in the testicular parenchyma
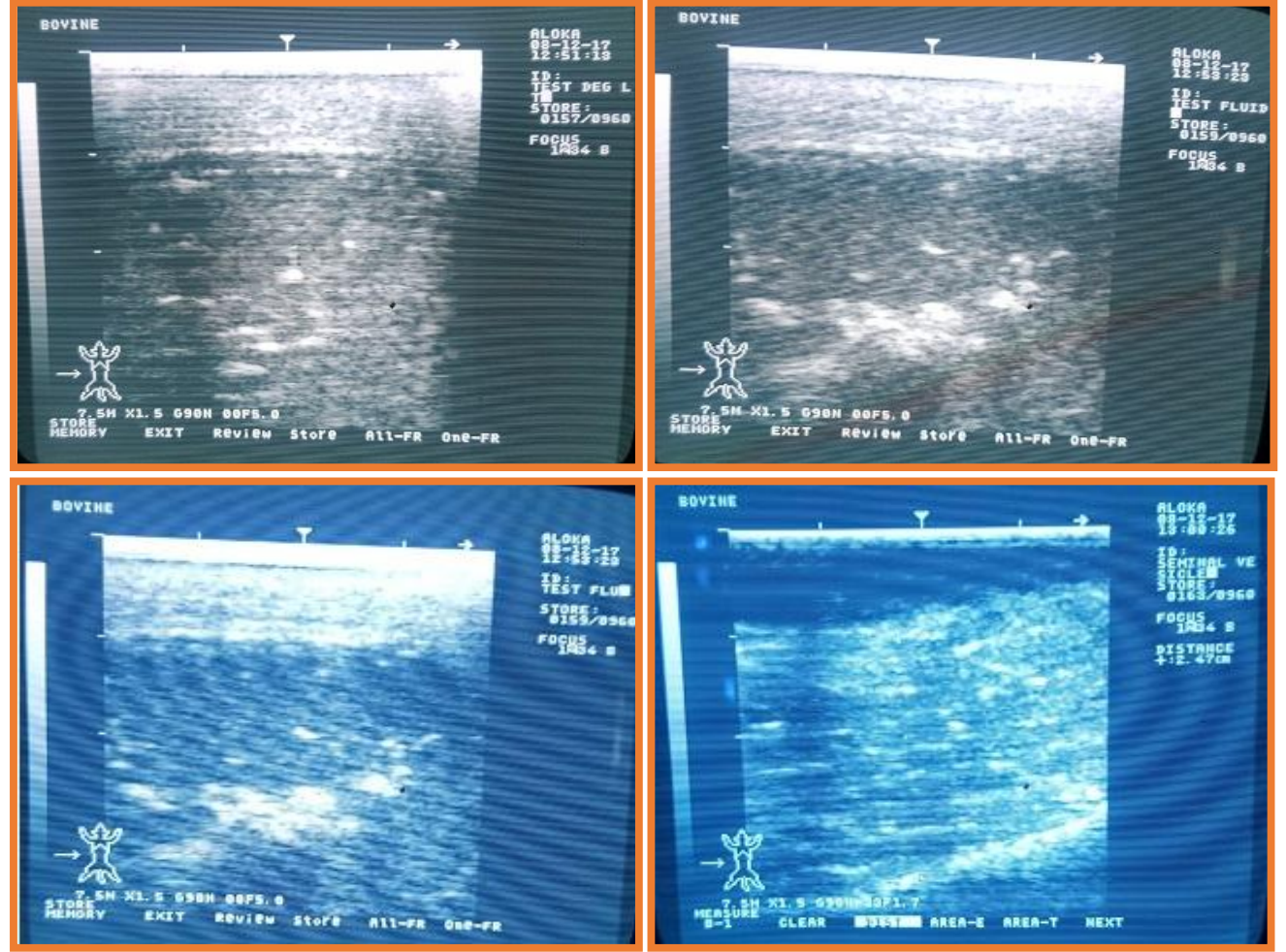

Also, extensive fibrosis is thought to reduce

\section{References}

the sperm production and may increase sperm abnormalities. Certain dietary factors such as deficiency of vitamins and minerals are also considered as a cause of testicular pathologies.

Barth, A.D., Alisio, L., Aviles, M., Arteaga, A.A., Campbell, J.R., Hendrick, S.H., 2008. Fibrotic lesions in the testis of bulls and relationship to semen quality. Animal Reproduction Science 
106: 274-288.

Inc., San Diego, CA, pp. 263.

Arteaga, A., Barth, A., Brito, L., 2005. Ramirez-Mendoza, H., Hernandez-Jauregui, Relationship between semen quality and pixel-intensity of testicular ultrasound images after scrotal insulation in beef bulls. Theriogenology 64: 408-415.

McEntee, K., 1990. Reproductive Pathology P., Reyes-Leyva, J., Zenteno, E., Moreno-Lopez, J., Kennedy, S., 1997. Lesions in the reproductive tract of boars experimentally infected with porcine rubulavirus. J. Comp. Pathol. of Domestic Animals. Academic Press

\section{How to cite this article:}

Bilawal Singh, Navgeet Singh, Ajeet Kumar, S.S. Dhindsa and Khushpreet Singh. 2019. Infertility in a Sahiwal Bull due to Bilateral Testicular Fibrosis. Int.J.Curr.Microbiol.App.Sci. 8(05): 1975-1978. doi: https://doi.org/10.20546/ijcmas.2019.805.229 